\title{
Modélisation de l'évolution à long terme de la flore adventice. I. Construction d'un modèle descriptif de l'évolution quantitative du stock de semences de l'horizon travaillé
}

\author{
Philippe DEBAEKE * (1) \& Michel SEBILLOTTE \\ * I.N.R.A., Laboratoire d'Agronomie, Centre de Recherches de Paris-Grignon-Massy, F 78850 Thiverval-Grignon \\ I.N.A., Chaire d'Agronomie, 16, rue Claude-Bernard, F 75231 Paris Cedex 05
}

Mots clés additionnels : Système de culture, désherbage. evolution of the seed bank in the cultivated layer.

The qualitative and quantitative composition of a local weed flora mainly reflects the existing cropping system; this observation concerns both seedlings and seed bank. In a crop rotation, the cropping system does not have rapid effects, especially for broad-leaved weeds, because the soil seed bank acts as a buffer. Till now, yearly records have been used to describe the incidence of cropping systems on the evolution of weed communities; but they do not account for the origins of annual changes and are unsuitable for prediction. The present work deals with the construction of a model describing the quantitative evolution of the soil seed bank in the cultivated layer and is better able to attain such objectives. This deterministic and discrete model simulates step by step the fate of a weed population in an annual crop and is founded on an annual statement of the seed content of the soil. Its recurrent application leads simply to a forecast of the long-term evolution of the weed flora. The limits of this approach are discussed : in its current version, the model is restricted to rather low weed densities.

Additional key words : Cropping system, weed control.

\section{INTRODUCTION}

Les modèles permettant de raisonner à court et (ou) à long terme la lutte contre les mauvaises herbes des cultures (et en premier lieu l'opportunité d'un désher-

(') Nouvelle adresse: I.N.R.A., Station d'Agronomie, Centre de Recherches de Toulouse, B.P. 27, Auzeville, F 31326 CastanetTolosan Cedex bage chimique) font encore largement défaut, du fait de la complexité des processus régissant l'évolution de la flore adventice, mais aussi de conditions économiques longtemps favorables aux traitements herbicides systématiques imposés par l'intensification. Or, la mise en évidence de changements qualitatifs et quantitatifs de la flore, sous l'effet des pratiques culturales, nécessite souvent des dispositifs de longue durée, coûteux et n'apportant pas de réponses en temps utile lorsqu'on- 
veut tester rapidement de nouveaux systèmes de culture ; la mise au point de modèles d'évolution de la flore adventice et leur utilisation en simulation permettraient de pallier ces insuffisances.

Des approches modélisées existent déjà dans ce domaine. Ainsi, à partir de situations expérimentales où tout apport de nouvelles semences est évité, ROBERTS (1970) propose une cinétique de décroissance exponentielle du stock semencier; le taux de déclin annuel est dans ce cas constant pour une espèce, une fréquence de travail du sol et un milieu donnés, indépendamment de l'âge des semences enfouies (ROBERTS \& FEAST, 1973). Cependant, ces conditions sont fort éloignées de la réalité du champ cultivé : les taux de déclin élevés (30 à 60 p. 100 par an, RoBERTs, 1970) sont peu compatibles avec le maintien de stocks semenciers importants dans les parcelles de grande culture régulièrement désherbées (Roberts, 1981; RoBerts \& ChANCEllor, 1986; Barralis \& Chadoeuf, 1987). En effet, dans ces conditions, les évolutions sont souvent lentes, notamment lorsque la rotation des cultures et des herbicides est pratiquee (BARRALIS \& CHADOEUF, 1976; BEURET, 1984; SCHWEIZER \& ZIMDAHL, 1984). Néanmoins, autour de la tendance, on enregistre des fluctuations inter-annuelles du stock non négligeables, liées à la variabilité du climat et à l'alternance des cultures (BARRalis \& Chadoeuf, 1976).

Afin de prendre en compte la dynamique annuelle de la flore adventice (notamment le retour au sol des semences produites dans la culture), d'autres approches se sont amorcées récemment : monospécifiques, elles ne concernent encore que les espèces à stock peu persistant, en particulier les graminées annuelles infestant les céréales (Alopecurus myosuroides: CUSSANS \& Moss, 1982 ; Avena fatua: SElman, 1970 ; RAUber \& Koch, 1975 ; Mortimer et al., 1978, 1980; WiLSON et al., 1984 ; Bromus sterilis: Pollard, 1982). Ces modèles s'appuient sur les progrès acquis en dynamique des populations (SAGAR \& MORTIMER, 1976; HARFER, 1977) et sur de nombreux résultats expérimentaux; néanmoins, la plupart des études de dynamique sont pratiquées en site non perturbé; plus rares sont les travaux permettant de traduire l'effet des pratiques culturales (en premier lieu le désherbage chimique) sur la démographie des populations naturelles (MANLOVE $e t$ al., 1982; JoHNSTON \& CoBle, 1986 ; RAI \& TRIPATHI, 1986 ; FERNANDEZ-QUINTANILLA et al., 1987).

Dans ce $1^{\mathrm{er}}$ article, un modèle d'évolution quantitative pluri-annuelle de la densité de semences adventices du sol est proposé ; cette construction tient compte de la dynamique annuelle de la flore et sa vocation est prévisionnelle (à moyen et long terme). Un $2^{\mathrm{c}}$ article concernera l'estimation des paramètres et la validation du modèle, dans le cas de dicotylédones annuelles à stock persistant.

\section{II. ÉTABLISSEMENT DU MODÈLE}

\section{A. Principe général : le bilan de semences}

La figure 1, inspirée de Cussans (1976) pour Avena fatua, présente les étapes du devenir annuel des semences du sol et de leur renouvellement. La probabilité de

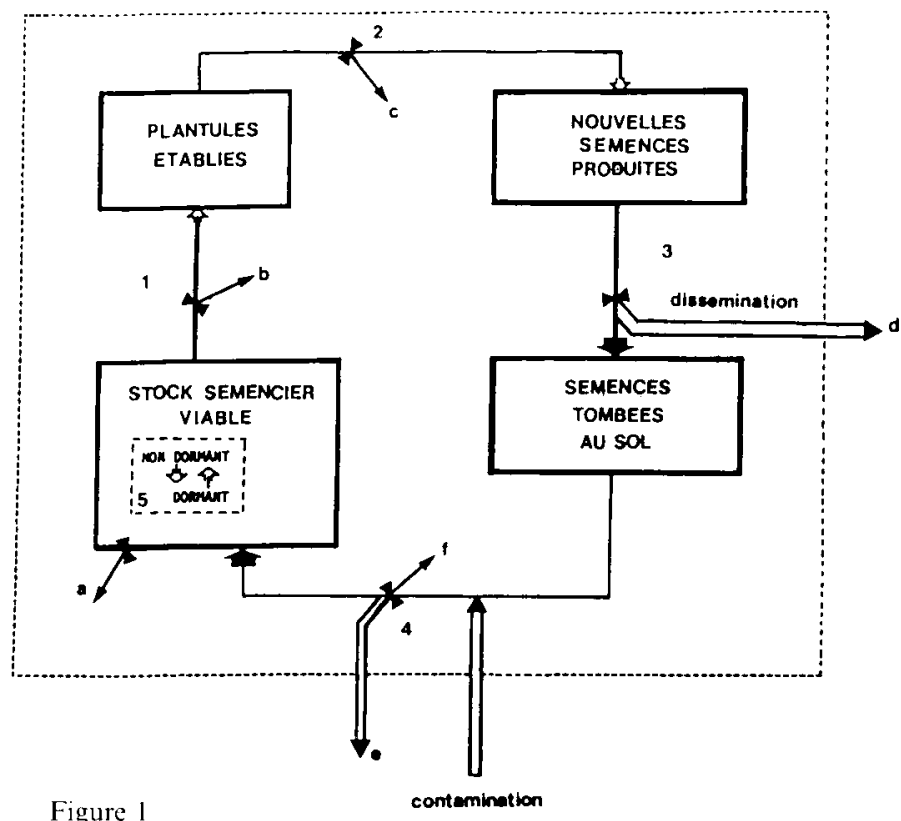

Représentation schématique de l'évolution du stock semencier d'un champ cultivé au conts du clele annuel dine espece adventice.

Etapes-cles du cycle $\square$

Interactions sol, climat. peaplenent cultivé, tecturiques $\mathbf{X}$

Phases du cycle : _. $\rightarrow$ changements d'état de la flore

1) Germination - levee.

2) Formation et maturation des semences.

3) Chute des semences.

4) Enfouissement des semences.

5) Erolution cuclique de la dormance.

Causes de pertes de semences: $\longrightarrow$ pertes internes

$\longrightarrow$ echanges de semences avec I'oxtérieur.

a) Perte de viahilité des semences enfouies non germées (prédation parasitisme, vicillissenent).

b) Eihec de la levée (semences trop profondes, sécheresse après germination, herbicides de pré-levée, prédation, parasitisme).

c) Mortalité de plantules (gel ombrage, herbicides de post-levée, prédation, parasitisme, travail du sol).

$d$, e) Exportation de semences (d: vent, produit récolté, engins de récolte : e : résidus de culture, paille notamment).

f) Destruction de semences e'n surface du sol (prédation, brülage).

Schematic represchation of the soil seed-bank elolution for an anmuat weed in a cultivated field

Mean stages of the crele $\square$

Interactions betwe'n soil dimate, canopy and crop management $\mathbf{X}$

Phase of the cycle: $\longrightarrow$ state changement of the flora

emergence.

2) Seed production and maturation.

3) Seed shedding.

4) Seed hurial

5) Cuclic evolution of dormancy.

Origins of the seed losses: $\longrightarrow$ internal loss $\longrightarrow$ seed exchanges.

a) Viability loss of the buricd ungerminated seeds (predation, parasi tism, ageing).

b) Failure of emergence (deeper secds, lack of moisture following germination, pre-emergence herbicides, predation, parasitism).

c) Plant mortality (frost, shading, post-emergence herbicides, predation, parasitism, (cultivation).

d) Secd exports (wind, crop, harvest machines).

e) Seed exports (crop residhes, mainly straw).

f) Superficial seed loss (predation, burning).

passage d'un stade à l'autre et le niveau de réalisation de chaque stade dépendent des conditions et facteurs du milieu résultant d'inter-relations entre le sol, le climat, la culture et les techniques appliquées. Des pertes de semences et de plantes sont enregistrées à chaque étape. Les limites du système concernent la couche de sol labourée et la parcelle, ce qui n'exclut pas des échanges 
de semences avec l'extérieur par les processus de dissémination (naturels ou liés aux techniques). Ce cycle se déroule en continu sur l'année et la plupart des états de la flore décrits à la figure 1 coexistent à un moment donné au sein d'une population (variabilité des états dormants, POPAY \& RoBERTS, 1970) ou d'une communauté adventice (variabilité interspécifique des pics de levée, Montegut, 1975). On s'attend à des comportements variables des plantes selon la position de leur cycle face à celui de la culture (effet de l'espèce et de la classe d'âge). Le maintien de son stock semencier dépendra pour une adventice de ses caractéristiques génétiques (production potentielle, persistance des semences) et de sa capacité à accomplir son cycle dans la culture (position du cycle par rapport aux événements climatiques et aux opérations culturales, tolérance aux herbicides et à la concurrence du peuplement cultivé). Un bilan de semences annuel contribuerait ainsi à l'estimation de l'effet précédent défini par SEBILLOTTE (1982) comme «la variation d'état du milieu entre le début et la fin de la culture sous l'influence combinée de la plante, des techniques culturales qui lui sont appliquées, l'ensemble étant soumis au climat ". L'itération de ce bilan sur plusieurs années devrait permettre de comprendre les évolutions de stock observées.

\section{B. Formulation du modèle}

\section{Bilan annuel}

A l'issue de l'année culturale $\mathrm{i}+1$, après retour au sol des semences produites dans la culture, le stock semencier parcellaire $\mathrm{Si}+1$ (i désignant l'année), sur l'épaisseur maximale de la couche perturbée par les outils, s'exprime de la manière suivante :

$$
\mathrm{S}_{\mathrm{i}+\mathrm{I}}=\mathrm{S}_{\mathrm{i}}-\mathrm{L}_{\mathrm{i}+\mathrm{l}} \cdot \mathrm{M}_{\mathrm{i}+\mathrm{1}}-\mathrm{E}_{\mathrm{i}+\mathrm{l}}+\mathrm{P}_{\mathrm{i}+1}+\mathrm{I}_{\mathrm{i}+\mathrm{l}}
$$

L’indice i désigne l'année d'estimation (stock) ou de réalisation de chaque poste (paramètres démographiques). $\mathrm{S}$ et $\mathrm{S}_{\mathrm{j}+1}$ s'expriment en nombre de semences bien conformées extérieurement, par $\mathrm{m}^{2}$ et sur l'horizon $0-30 \mathrm{~cm}$.

Les pertes de semences pour le système sont constituées par:

- le nombre de plantes levées $\left(\mathrm{L}_{\mathrm{i}+1}\right)$;

- le nombre de semences mortes dans le sol ou en surface sans donner de plantules viables ou décelables $\left(\mathrm{M}_{\mathrm{i}+1}\right)$; il s'agit ici de disparitions in situ et occultes ;

- le nombre de semences produites sur la parcelle et exportées $\left(E_{i+1}\right)$.

\section{Les apports concernent :}

- le nombre de semences produites sur la parcelle, tombées au sol et incorporées au stock $\left(\mathrm{P}_{\mathrm{i}+1}\right)$;

- le nombre de semences d'origine extérieure au système et importées au cours de l'année $\left(\mathrm{I}_{\mathrm{i}+\mathrm{l}}\right)$.

Hormis $I_{i+1}$, chaque poste du bilan peut s'exprimer comme une fraction du stock initial $\mathrm{S}_{\mathrm{i}}$, dont il est issu ; ainsi, on pose :

$$
\mathrm{L}_{\mathrm{i}+1}=\alpha_{\mathrm{i}+1} \cdot \mathrm{S}_{\mathrm{i}}
$$

$\alpha_{i, 1}$ est le taux annuel de levée

$$
\mathrm{M}_{\mathrm{i} 11}=\gamma_{\mathrm{i}+\mathrm{I}} \cdot \mathrm{S}_{\mathrm{i}}
$$

$\gamma_{\mathrm{i}+\text { I }}$ est le taux annuel de mortalité des semences in situ

$$
E_{i+1}=\alpha{ }_{i+1} \cdot\left(1-\sigma_{i+1}\right) \cdot \varepsilon_{i+1} \cdot p_{i+1} \cdot S_{i}
$$

$\mathrm{P}_{\mathrm{i}+1}=\alpha{ }_{\mathrm{i}+1} \cdot\left(1-\sigma_{\mathrm{i}+1}\right) \cdot\left(1 \quad \varepsilon_{\mathrm{i}, 1}\right) \cdot \mathrm{p}_{\mathrm{i}+1} \cdot \mathrm{S}_{\mathrm{i}}$

$\alpha{ }^{\prime \prime}{ }_{\mathrm{i}+1}$ est la fraction du stock germant et levant du semis à la récolte de la culture

$\sigma_{i+1}$ est le taux de mortalité annuel des plantules établies, avant production de semences

.$\varepsilon_{\mathrm{j}+1}$ est la fraction exportée de la production semencière autochtone

$\mathrm{p}_{\mathrm{i}+\text {, }}$ est le nombre de semences bien conformées par plante présente à la récolte.

$I_{i+1}$ est considéré dès maintenant comme marginal devant le stock $\mathrm{S}_{\mathrm{i}}$; nous reviendrons plus loin sur les conditions de validité de cette hypothèse simplificatrice.

Dans ce contexte, l'expression du bilan annuel prend la forme suivante:

$$
\begin{aligned}
S_{i+1}= & S_{i}\left[1-\alpha_{i+1}-\gamma_{i+1}\right. \\
& -\left[\alpha_{i+1}^{\prime \prime} \cdot\left(1-\sigma_{i+1}\right) \cdot \varepsilon_{i+1} \cdot p_{i+1}\right] \\
& \left.+\left[\alpha_{i+1}^{\prime \prime} \cdot\left(1-\sigma_{i+1}\right) \cdot\left(1-\varepsilon_{i+1}\right) \cdot p_{i+1}\right]\right](6)
\end{aligned}
$$

Chaque paramètre a une valeur comprise entre 0 et 1 .

\section{Effet des classes d'âge (ou cohortes)}

La période de levée d'une plante est souvent déterminante pour sa survie et sa fécondité, qu'il s'agisse de la concurrence exercée par la culture ou les adventices déjà installées, de l'effet d'une opération culturale (désherbage, travail du sol) ou de l'action du climat (gel, sécheresse, photopériode) (HARPER, 1977). L'étalement des dates de maturation et de libération des semences qui en résulte (LEGUIZAMON \& ROBERTS, 1983) risque également d'affecter le retour au sol des semences. Aussi est-il nécessaire de retenir des valeurs différentes des paramètres $\alpha^{\prime \prime}, \sigma, \varepsilon, p$ selon les classes d'âge, après regroupement des cohortes à comportement peu tranché.

L'expression (6) devient alors :

$$
\begin{aligned}
& \mathrm{S}_{\mathrm{i}+1}=\mathrm{S}_{\mathrm{i}}\left[1-\alpha_{\mathrm{i}+1}-\gamma_{\mathrm{i}+1}\right. \\
& \quad-\sum_{j=1}^{m}\left[\alpha_{i+1, j}^{\prime \prime} \cdot\left(1-\sigma_{i+1, j}\right) \cdot \varepsilon_{\mathrm{i}+1, j} \cdot \mathrm{p}_{\mathrm{i}+1, j}\right] \\
& \left.\quad+\sum_{\mathrm{j}=1}^{m}\left[\alpha_{i+1, j}^{\prime \prime} \cdot\left(1-\sigma_{i+1, j}\right) \cdot\left(1-\varepsilon_{\mathrm{i}+1, j}\right) \cdot \mathrm{p}_{\mathrm{i}+1, j}\right]\right]
\end{aligned}
$$

L'indice j se rapporte à la cohorte, par ordre de levée.

\section{Bilan pluri-annuel}

Si on définit un point origine par le stock $S_{0}$, partant de l'équation (7), on obtient par récurrence, après $\mathrm{n}$ années culturales, l'expression :

$$
\mathrm{S}_{\mathrm{n}}=\mathrm{S}_{\mathrm{o}} \cdot \mathrm{K}_{\mathrm{n}}
$$


Effets prévisibles des techniques de récolte et de post-récolte sur le devenir des semences adventices produites dans la culture: cas d'un blé d'hiver et de 3 dicotylèdones annuelles fréquentes.

Predicted effects of harvest and post-harvest management on the fate of weed seed production : case of winter wheat and 3 frequent broad-leaved weeds.

\begin{tabular}{|c|c|c|c|}
\hline $\begin{array}{l}\text { Date } \\
\text { de libération } \\
\text { des semences } \\
\text { adventices }\end{array}$ & $\begin{array}{l}\text { En majorité } \\
\text { avant la récolte }\end{array}$ & \multicolumn{2}{|c|}{$\begin{array}{c}\text { Etaléc } \\
\text { ou en majorité } \\
\text { après la récolte }\end{array}$} \\
\hline $\begin{array}{l}\text { Hauteur } \\
\text { de la plante } \\
\text { à la récolte }\end{array}$ & & $\begin{array}{l}\text { Inférieure } \\
\text { à la hauteur } \\
\text { de coupe }\end{array}$ & $\begin{array}{l}\text { Supérieure } \\
\text { à la hautcur } \\
\text { de coupe }\end{array}$ \\
\hline $\begin{array}{l}\text { Conséquences } \\
\text { de la récolte } \\
\text { sur le devenir } \\
\text { des semences }\end{array}$ & $\begin{array}{c}\text { La récolte joue peu } \\
\text { sauf si elle est avancée } \\
\text { (ensilage) } \\
\text { pour les espèces hautes }\end{array}$ & $\begin{array}{c}\text { La récolte joue peu } \\
\text { sur ces espèces } \\
\text { basscs } \\
\text { ou à port étalé }\end{array}$ & $\begin{array}{l}\text { La récolte: } \\
\text { - stoppe la formation } \\
\text { des semences viables } \\
\text { - cxporte dans le grain } \\
\text { des semences } \\
\text { - modific la répartition } \\
\text { parcellaire des } \\
\text { semences (rejet avec les balles) }\end{array}$ \\
\hline $\begin{array}{l}\text { Effet } \\
\text { des techniques } \\
\text { de post-récolte }\end{array}$ & $\begin{array}{l}\text { Le brûlage des résidus } \\
\text { peut réduire le nombre } \\
\text { de semences viables }\end{array}$ & $\begin{array}{l}\text { Le déchaumage avant } \\
\text { formation ou maturation } \\
\text { des semences et le brûlage } \\
\text { peuvent réduire le nombre } \\
\text { de semences viables }\end{array}$ & $\begin{array}{l}\text { L'exportation ou le brûlage } \\
\text { de la paille réduisent } \\
\text { le nombre de semences viables }\end{array}$ \\
\hline $\begin{array}{l}\text { Conséquences } \\
\text { pour } E_{i}: 1\end{array}$ & Valeurs faibles à nulles & Valeurs faibles à nulles & $\begin{array}{l}\text { Valeurs variables selon } \\
\text { le devenir des pailles } \\
\text { et la qualité du tri } \\
\text { par la moissonneuse-batteuse }\end{array}$ \\
\hline Exemples & $\begin{array}{c}\text { Capsella } \\
\text { bursa-pastoris }\end{array}$ & $\begin{array}{l}\text { Anagallis } \\
\text { arvensis }\end{array}$ & $\begin{array}{l}\text { Fallopia } \\
\text { convolvulus }\end{array}$ \\
\hline Type & I & II & III \\
\hline
\end{tabular}

avec

$$
\mathrm{K}_{\mathrm{n}}=\prod_{\mathrm{i}=1}^{\mathrm{n}} \mathrm{k}_{\mathrm{i}} \quad \text { et } \quad \mathrm{k}_{\mathrm{i}}=\mathrm{S}_{\mathrm{i}} / \mathrm{S}_{\mathrm{i}-1}
$$

$\mathrm{k}_{\mathrm{i}}$ est le taux de croissance annuel de la population de semences d'une parcelle au cours de l'année $i$ et $K_{n}$ désigne alors le taux de croissance cumulé correspondant, après $\mathrm{n}$ années d'application d'un système de culture.

Le calcul de $S_{n}$ s'effectue de manière automatisée à l'aide d'un programme Basic, à partir d'une matrice composée du stock initial et des valeurs annuelles de chaque paramètre; celles-ci tiennent compte des effets cumulatifs. Des sorties annuelles et cumulées sont fournies par le programme.

\section{JUSTIFICATION DES LIMITES DU SYSTEME ET DU CHOIX DES PARAMÈTRES}

\section{A. Limites du système}

\section{La couche travaillée}

L'horizon $0-30 \mathrm{~cm}$ constitue la zone d'accumulation quasi exhaustive des semences incorporées par le travail du sol. Une colonisation plus profonde peut avoir lieu : labour profond (ROBERTS, 1963), ingestion par les vers de terre (MCRILL \& SAGAR, 1973), pénétration des petites semences par la fissuration estivale du sol (Robinson \& KUST, 1962). Ces processus sont peu quantifiés; la décroissance de la densité de semences avec la profondeur, observée en sol travaillé ou non (ROBERTS, 1981), nous autorise à négliger d'éventuelles migrations vers le sous-sol.

\section{La parcelle}

Il est peu raisonnable de considérer la parcelle (surface de sol traitée de manière homogène) comme un système parfaitement fermé aux échanges de semences avec l'extérieur. Cependant on peut considérer le stock semencier de la parcelle comme l'origine essentielle des infestations de surface lorsque la valeur des postes $E_{i+1}$ et $\mathbf{I}_{i+1}$ est négligeable en regard respectivement de la production annuelle restituée $P_{i+1}$ et du stock initial $S_{i}$ (hypothèse hl). Il est possible que pour une espèce donnée les quantités de semences exportées et importées puissent se compenser annuellement soit $\mathrm{I}_{\mathrm{i}+1}-\mathrm{E}_{\mathrm{i}+1}=$ 0 dans l'expression (1) (hypothèse 2). Examinons la validité de ces hypothèses simplificatrices.

\section{a) La contamination}

Le poste $I_{i \vdash 1}$ est composé de semences importées par les techniques culturales (travail du sol, semis, irriga- 
tion, amendements divers...) et par dissémination naturelle (vent). Si la contamination par les semences de mauvaises herbes des lots de graines semées a pu constituer une source importante de maintien des stocks et de propagation de certaines espèces, l'utilisation actuelle de lots criblés et certifiés, mais aussi le meilleur contrôle par les herbicides de la production de semences adventices viables, conduisent à de faibles taux d'impuretés sinon dans les lots récoltés du moins dans les lots semés (Tonkin \& Phillipson, 1973 ; Barralis, 1982). La dissémination naturelle des semences sur de longues distances est limitée à quelques espèces munies d'organes adaptés ; c'est le cas des Composées qui constituent l'essentiel des semences apportées par le vent (HARPER, 1977 ; Froud-WiLliams et al., 1981 ; ARCHIBOLD \& Hume, 1983). Pour les autres espèces et même dans le cas de semences légères, une chute autour du pied-mère est la situation la plus fréquente surtout sous couvert d'une culture (SAliSbURY, 1961; MAJOR \& PYOTT, 1966; Hurka \& HaAse, 1982). Cette tendance est confirmée par les cartographies parcellaires, qui soulignent la constance à long terme de la répartition initiale des infestations et donc les faibles mouvements de semences extra-parcellaires (CHANCELLOR, 1976; HuRKa \& HAASE, 1982). Des contaminations lors d'irrigations ou d'épandages de boues ou fumier ont été signalées de manière peu chiffrées; si l'on excepte ces systèmes particuliers (élevage, cultures irriguées) et les espèces à dissémination aérienne, on peut conclure raisonnablement à de très faibles valeurs de $I_{i+1}$ devant le stock initial $S_{i}$ (hypothèse 1 ).

\section{b) L'exportation}

Celle-ci se réalise pour l'essentiel avec le produit récolté ou les résidus de culture non enfouis. Ces pertes dépendent de la période de libération des semences et de la hauteur des plantes adventices lors de la récolte; à partir de ces deux critères, il est alors possible de raisonner les risques d'exportation selon les espèces adventices. Cette démarche est illustrée au tableau 1 dans le cas des céréales et plus généralement des cultures récoltées à la moissonneuse-batteuse ou avec une barre de coupe. Trois types de plantes ont été distingués. Pour les 2 premiers, on est en droit d'admettre une faible valeur de $E_{i+1}$ devant $P_{i+1}$ (hypothèse 1 ), comme le prouvent a contrario les études de pureté des lots de céréales récoltées (WELlington, 1960 ; TONKIN \& PHILLiPson, 1973 ; Pawlowski \& Wszolek, 1978). Pour les plantes à port dressé ou grimpant du type III, dont les semences sont à divers stades au moment de la récolte mais non libérées dans l'ensemble, le risque d'exportation dépendra de la taille ou du poids des semences, de la qualité du tri opéré par la moissonneuse et du devenir de la paille. Pour les petites semences, le rythme de rejet des impuretés conditionne les échanges de semences entre parcelles et la répartition intra-parcellaire des semences de l'année. Ce poste n'est pas négligeable pour les graminées annuelles ou certaines dicotylédones comme Galium aparine: par leur taille et leur cycle coïncidant avec celui de la céréale, ces espèces se montrent très sensibles aux modalités de récolte (date notamment), ce qui se traduit par des valeurs de $E_{i+1}$ très variables entre parcelles et entre années (WILSON, 1970 ; Moss, 1983). Pour les cultures arrachées (bette- rave sucrière), il faudrait tenir compte des semences entrainées avec la terre adhérant aux racines. Seule une gamme de données expérimentales permettrait d'évaluer l'amplitude des pertes; aussi, pour les espèces du groupe III, l'application du modèle est délicate.

Une compensation annuelle entre semences introduites et exportées (h2) n'est pas exclue dans certains cas (ruissellement, terre collée aux outils, migration latérale par le labour, dissémination par le vent); ces flux ne sont pas quantifiés ; cependant, s'ils assurent l'introduction de nouvelles espèces, il est peu probable qu'ils modifient de manière sensible la dynamique quantitative du stock semencier.

\section{L'année culturale}

Celle-ci démarre dès la première opération mécanique ou chimique suivant la récolte (le plus souvent un déchaumage superficiel) ; cette date marque l'enfouissement des semences, libérées ou non, produites sur le précédent, et (ou) la destruction de plantes à des états variés de leur développement. L'estimation de la production de semences mûres à cette époque (ou pouvant le devenir dans le sol) est très délicate, notamment pour les espèces du type II. Cet aspect sera abordé plus loin (B-4).

$\mathrm{Si}$ les références abondent en ce qui concerne la persistance des herbicides et leur toxicité pour la culture suivante, leur action résiduelle sur la flore adventice est mal connue. Cet effet pourrait affecter les levées automnales après un maïs traité aux triazines. Notre modèle ne considère pas d'éventuels effets bisannuels d'un herbicide même persistant.

\section{B. Justification des paramètres d'évolution interne}

\section{Taux de levée annuel}

Ce paramètre désigne le rapport du nombre de plantules visibles au champ (par $\mathrm{m}^{2}$ ), levant au cours de l'année culturale en zone non désherbée, au nombre initial de semences enfouies bien conformées (par $\mathrm{m}^{2}$, sur $0-30 \mathrm{~cm}$ ). Sa valeur est souvent faible mais néanmoins variable selon l'espèce (taille des semences, période de germination face au semis de la culture), la distribution verticale et l'àge des semences, l'état structural de surface (travail du sol), enfin le climat (pluviométrie) (Roberts, 1981; Debaeke, 1988a). Ce taux global se décompose en deux fractions :

- $\alpha^{\prime}$ concerne la flore levée dans l'interculture (déchaumage-semis); dans les conditions de grande culture, les plantules apparues au cours de cette période sont détruites par le labour avant production de semences. Dans le cas d'hivers très doux et de cultures semées tardivement au printemps, cette fraction pourrait avoir de l'importance sur le renouvellement du stock.

- $\alpha$ " se réfère aux plantules levant dans la culture; nous considérons que, dans la plupart des cas, seule cette fraction est à l'origine de nouvelles semences.

Une valeur moyenne de $\alpha_{i+1}$ est obtenue pour chaque combinaison " espèce adventice $\times$ date de semis de la culture $\times$ type de travail du sol $\times$ pluviométrie annuelle » à partir de situations où la distribution verticale des 
semences est assez régulière. Or, après labour, l'essen $t$ iel de la production de l'année $\mathrm{i}$ est enfouie entre 10 et $20 \mathrm{~cm}$ (Pawlowski \& Malicki, 1968 ; Chadeuf $e t$ al., 1984 ; DEBAEKE, 1988a) et contribue peu à la levée l'année $\mathrm{i}+1$. Aussi, chaque année, on tient compte de cet effet en multipliant la valeur moyenne de $\alpha_{i+1}$ par le terme $\left(1-\mathrm{P}_{\mathrm{i}} / \mathrm{S}_{\mathrm{i}}\right)$; une forte production de semences sur le précédent $\left(\mathrm{P}_{\mathrm{i}}\right)$ se traduit après labour par une localisation préférentielle des semences en profondeur et donc par une réduction du taux de levée $\left(\alpha_{i+1}\right)$. Cette pondération limite l'impact des fortes productions de semences. En l'absence de toute production, on considère que la distribution des semences n'est pas sensiblement modifiée, ce qui est cohérent avec les résultats d'expérimentation (DEBAEKE, 1987).

L'effet de l'âge des semences sur leur capacité germinative n'a pas été retenu dans ce modèle, les résultats étant fort divergents à ce niveau; alors que pour certains auteurs les semences âgées seraient plus dormantes (TAYLORSON, 1970 ; STOLLER \& WAX, 1974 ; Chadeur et al., 1984), Roberts \& FeAst (1973) montrent que le taux de levée ne varie pas avec l'âge du stock. Une différence se manifeste surtout entre semences produites sur le précédent et âgées de plus d'un an (ROBERTS, 1970 ; WiLSON et al., 1984).

\section{Taux de mortalité des semences in situ}

La durée de vie des semences enfouies est très variable et parfois prolongée (ROBERTS \& FEAST, 1973). Les causes de disparition sont diverses et mal connues: prédation, parasitisme, vieillissement physiologique, germination non suivie de levée ; ce dernier poste semble cependant le plus important (ROBERTS, 1972). Les différences spécifiques sont attribuables d'une part à la dureté des enveloppes et à l'existence d'une dormance cyclique (survie in situ) d'autre part à la taille des semences (réussite de la levée) (ROBERTS, 1970; TAYlorson, 1970; StOller \& WAX, 1974). Les déterminations de $\gamma_{i+1}$ restent néanmoins limitées à quelques milieux et à des conditions fort éloignées de celles du champ cultivé (enfouissements artificiels de semences fraîches, remuage fréquent du sol) ; la variable d'étude est dans ce cas le taux de disparition global du stock incluant les pertes par levée. Cependant, pour des populations d'àge varié, toutes espèces confondues, ce taux oscillerait entre 15 et 25 p. 100 (ROBERTS \& DaWkins, 1967 ; Roberts \& FEAST, 1973 ; Chadeuf $e t$ al., 1984).

On considère que pour une population d'âge varié la somme $\alpha+\gamma$ reste constante: après enfouissement profond de la production semencière $\mathrm{P}_{\mathrm{i}}$, on observe une réduction du taux de levée $\alpha_{i+1}$ (cf. B.l) et une hausse du même ordre de la mortalité in situ $\gamma_{i_{1}}$. La concentration des semences au-delà de $10 \mathrm{~cm}$ n'entraîne pas d'effets différentiels liés à la taille des semences, les profondeurs de levée maximales étant en majorité infërieures (Froud-Williams et al., 1984). Comme cela a été évoqué plus haut, on ne considère pas d'effet de l'âge des semences sur le taux de germination et donc sur le taux de mortalité par échec de la levée. L'enfouissement de semences peu ou pas dormantes se traduit probablement par un surcroît de mortalité qu'il est difficile de dissocier de l'effet profondeur lui-même.

On retiendra, pour chaque espèce, une valeur moyenne de $\gamma$, soumise chaque année à la correction précédemment évoquée. Cette valeur de référence, issue de la littérature, correspond à une population d'âge varié, distribuée de manière uniforme sur la couche de sol. Les facteurs de variation liés aux techniques culturales ne sont pas clairement identifiés; le travail du sol pourrait modifier sensiblement la valeur de ce poste (RoberTs \& DAWKINS, 1967 ; RoBERTS \& FEAST, 1973).

\section{Taux de mortalité des plantules}

\section{a) Origine des pertes}

Au cours du cycle cultural, les pertes de plantules sont souvent importantes, même en l'absence de désherbage, et les périodes de disparition bien délimitées (BARRALIS \& Chadeuf, 1980 ; Debaeke, 1988h). La construction du modèle prend implicitement en compte la destruction mécanique des plantes par le travail du sol $\left(\alpha_{i+1}^{\prime}\right.$ intervient comme perte, $p_{i+1}$ tient compte des plantes détruites par déchaumage avant production de semences). On considère que tout passage d'outil détruit complètement la flore présente. Les repousses de plantes enfouies par le labour sont rares; par contre, après travail superficiel au printemps, l'enfouissement peut être déficient, car le reverdissement du labour est parfois important (hiver doux, semis tardif) (MARTY et al., 1971). Seules des espèces à croissance rapide comme Stellaria media ou Poa annua peuvent former une masse difficile à enfouir ou capable d'une reprise végétative, en l'absence d'inversion complète de la couche de sol (ROBERTS, 1963 ; SOBEY, 1981).

Trois causes de disparition, intervenant souvent de manière consécutive, définissent la valeur de $\sigma_{i+1}$ :

- $\mu_{i+1}$ concerne la mortalité hivernale sous l'effet direct ou non du gel (adventices à levée automnale, cultures d'hiver) ;

- $\beta_{i+1}$ désigne la mortalité des plantes (et des semences germées) résultant de l'action des herbicides de postet pré-levée et du binage mécanique en cours de végétation;

- $\delta_{i+1}$ représente la mortalité printanière liée à la concurrence de la culture et des adventices (ombrage) mais aussi à la sécheresse du sol ;

Selon la date de semis de la culture, le type et le nombre des désherbages, $\sigma_{i+1}$ résulte de combinaisons diverses de ces paramètres (tabl. 2). En culture d'hiver, la concomittance des effets « herbicide de pré-levée » et " gel » nous oblige à retenir la valeur maximale de ces deux termes; il en va de même lorsque les actions des herbicides de pré- et post-levée se recoupent. Aucune interaction n'est admise entre ces pertes dans un tel modèle déterministe : pas de sensibilité différentielle aux herbicides et à la concurrence de la culture des plantes ayant survécu au gel, à la sécheresse ou à un désherbage antérieur. L'efficacité de l'herbicide n'est pas affectée également par la densité de peuplement ou les conditions d'éclairement au sol. En peuplement dense ou pour de faibles éclairements, l'action de l'herbicide apparaît pourtant renforcée (HAMMERTON, 1970; ERVIO, 1977 ; RAI \& TRIPATHI, 1986) ; la littérature est trop réduite à ce sujet pour accorder une portée générale à ces résultats. 


\section{b) Mortalité hivernale}

De précédents travaux ont montré qu'il existait au sein d'une communauté adventice des tolérances très variables au gel (RADEMACHER, 1964; SOBEY, 1981; DeBAEKe, 1988b). Celle-ci est liée au stade atteint par les plantes au moment du gel ou à des processus physiologiques. Des taux constants seront retenus pour les plantes à comportements tranchés (espèces peu ou très sensibles). Lorsque la mortalité des individus est liée à leur stade (croissance ou développement) et que celui-ci est variable lors du gel, une relation empirique entre $\mu$ et la somme de températures efficaces sur la période semis de la culture-gel doit être recherchée, comme cela a été proposé pour Capsella bursa-pastoris (DEBAEKE, 1988b). Cette valeur tient compte de la date de semis et du climat automnal, mais non de l'intensité et de la durée de la période gélive. La base thermique retenue pour le calcul (exigences minimales pour la germination, la croissance et le développement) est estimée de manière empirique ou se référe à la bibliographie disponible.

\section{c) Mortalité liée au désherbage}

Au cours de la culture, le programme de désherbage associe interventions chimiques (1 à 3 ) et mécaniques. Chaque opération se traduit par une efficacité et une persistance potentielles, mais aussi par une régularité d'action. Le taux de destruction réel dépend des techniques de pulvérisation et des conditions de sol et de climat au moment du traitement et sur la période de rémanence du produit, mais aussi de l'état de la population de mauvaises herbes (sensibilité liée au stade). La courbe de levée de la flore adventice est indispensable pour raisonner le taux de destruction annuel. Si la périodicité de germination est marquée et peu variable entre années pour certaines espèces (MONTEGUT, 1975). on observe pour d'autres, aux exigences plus souples, un profil de levée davantage affecté par le climat de l'année (ROBERTS \& POTTER, 1980). A partir de résultats expérimentaux donnant la répartition mensuelle de la levée au champ sur plusieurs années et en l'absence de désherbage (DeBAeke, 1988b), il est possible de relier cette courbe aux sommes de températures efficaces (sur les périodes où l'humidité du sol n'est pas jugée limitante) ; des approches similaires ont d'ailleurs été abordées pour d'autres annuelles (PSARSKI et al., 1986). En première approche, on retient les températures minimales de germination fournies par les auteurs $(\mathrm{KoCH}$, 1970 ; Montegut, 1975). Il est alors possible de prévoir chaque année la part de la levée annuelle présente au moment du traitement et sur la période d'action de l'herbicide. Les nombreuses références qualitatives et quantitatives découlant des essais de comportement herbicide (Anon., 1983, 1987) permettent de définir pour chaque herbicide un taux de destruction moyen des plantes présentes, par espèce et par stade de développement, ainsi qu'une persistance d'action (ponctuelle pour les produits à absorption foliaire, prolongée pour les herbicides à absorption racinaire); ces données manquent cependant de gammes de variation. La confrontation à chaque instant de la flore présente (définie par une contribution à la levée totale et une distribution des stades) et de l'herbicide (défini par un taux de destruction et une durée d'action) permet d'aboutir in fine au taux de destruction total $\beta_{i+1}$. A titre d'exemple, la figure 2 illustre le calcul de $\boldsymbol{\beta}_{i+1}$ dans le cas d'une espèce indifférente et d'une culture d'hiver.

Le climat n'intervient ici que pour déterminer les plantes soumises à l'herbicide et n'est pas retenu comme facteur de variation de l'activite du produit lors de l'application ou peu après, ni de sa vitesse de dégradation dans le sol. Malgré des approches récentes (WALKer \& BARNES, 1981), la cinétique d'évolution des résidus herbicides reste difficile à prévoir au champ, aussi nous retiendrons un taux de destruction constant sur la durée moyenne de persistance du produit. Ainsi, les facteurs de variation de $\beta_{i+1}$ concernent l'espèce, la nature de l'herbicide, la répartition saisonnière de la levée (liée au climat) et le stade de développement des adventices traitées.

\section{d) Mortalité printanière}

Le poste $\delta_{i+1}$ désigne les pertes de plantules (surtout printanières) sous couvert de la culture. Il s'agit dans ce cas d'une interaction entre prédation, parasitisme, sécheresse et manque de lumière (SAGAR \& MorTimer,

TABLEAU 2

Mode de calcul de $\sigma$, taux de mortalité annuel total de la flore levée, en fonction du programme de désherbage ét de l'époque de semis de la culture. Calculation of the $\sigma$ parameter, annual plant mortality rate, according to the weed control program and crop seeding rate.

\begin{tabular}{|c|c|c|c|c|c|}
\hline Epoque & $\begin{array}{c}\text { Programme } \\
\text { de } \\
\text { désherbage }\end{array}$ & Pré-levée & Post-levée & $\begin{array}{c}\text { Pré-lcvée } \\
+ \\
\text { post-levée }\end{array}$ & Impasse \\
\hline Hiver & & $\mathrm{M}\left(\mu, \beta_{1}\right) \cdot \delta$ & $\mu \cdot \beta_{2} \cdot \delta$ & {$\left[M\left(\mu, \beta_{1}\right) \cdot M\left(\beta_{1}, \beta_{2}\right) \cdot \beta_{2} \cdot \delta\right]$} & $\mu \cdot \grave{\delta}$ \\
\hline Printemps & & $\beta_{1} \cdot \delta$ & $\beta_{2} \cdot \delta$ & $M\left(\beta_{1}, \beta_{2}\right) \cdot \beta_{2} \cdot \delta$ & $\delta$ \\
\hline
\end{tabular}

M : Maximum des deux valeurs (sur la période où les deux causes de mortalité se superposent).

$\beta_{1}$ : taux de mortalité lié à l'herbicide de pré-levée.

$\beta_{2}$ : taux de mortalité lié à l'herbicide de post-levée.

$\mu$ : taux de mortalité hivernale.

$\delta$ : taux de mortalité par ombrage 


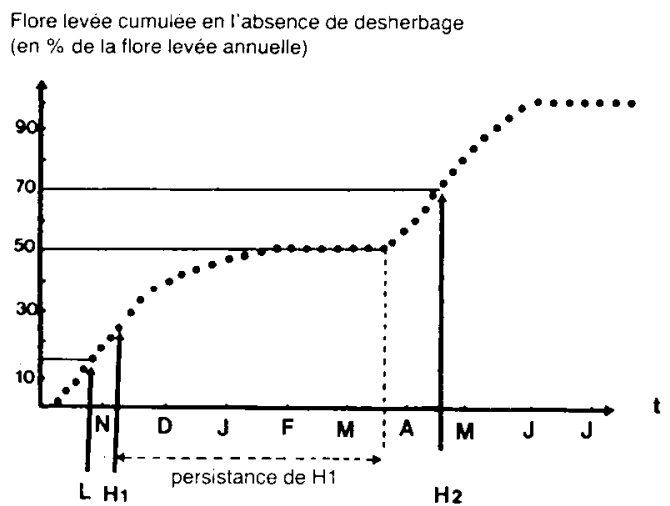

\begin{tabular}{|c|c|c|c|}
\hline $\begin{array}{l}\text { Nature } \\
\text { de } \\
\text { l'intervention }\end{array}$ & $\begin{array}{l}\text { Flore présente } \\
\text { au moment } \\
\text { de l'intervention } \\
\text { et sur sa période } \\
\text { d'action } \\
\text { (en \% de la flore } \\
\text { levéc annuelle) }\end{array}$ & $\begin{array}{c}\text { Efficacité } \\
\text { de l'intervention } \\
(\% \text { de destruction } \\
\text { de la flore } \\
\text { présente) }\end{array}$ & $\begin{array}{l}\text { Part } \\
\text { de la levé } \\
\text { annuelle } \\
\text { détruite }\end{array}$ \\
\hline Labour (L) & $x=15$ & 100 & 15,0 \\
\hline $\begin{array}{c}\text { Herbicide } \\
\text { de pré-levée } \\
\left(\mathrm{H}_{1}\right)\end{array}$ & $y \quad x=35$ & 90 & 31.5 \\
\hline $\begin{array}{l}\text { Herbicide } \\
\text { de post-levée } \\
\left(\mathrm{H}_{2}\right)\end{array}$ & $\begin{array}{c}0,1(y-x)=3,5 \\
\text { levées automnales } \\
\text { non détruites par } H_{1} \\
z-y=20 \\
\text { levées printanières } \\
\text { préscntes au moment de } H_{2}\end{array}$ & 80 & 19,0 \\
\hline
\end{tabular}

Total annucl : 68.3

$68 \%$ de la flore levée annuclle disparaît dans ce cas sous laction conjuguée du travail du sol d'implantation et des herbicides, soit $\beta=$ 0,68

La courbe de levée et les taux d'efficacité sont théoriques.

Figure 2

Mode de calcul du paramère $\beta$, taux de mortalité annuel par désherhige de la flore levée : exemplo théorique d'une'culture semée à l'automn' at d'une adventice à levée èchelonnée.

Calculation of the $\beta$ parameter : case of an autumn-seeded crop and a weed with staggered emorgenc'

1976); en culture de céréales, l'ombrage apparaît comme la cause essentielle de mortalité (FogelFors, 1977 ; DEBAEKE, 1988b).

La courbe de levée indexée au climat, évoquée plus haut, permet de déterminer la contribution de chaque classe d'âge et de lui affecter un taux de mortalité particulier. Sur la base de résultats antérieurs (DEBA.E$\mathrm{KE}, 1988 b$ ), on retiendra 3 cohortes en culture d'hiver (1. levées d'automne, 2. levées de mars-avril, 3. levées de mai-juin), deux en culture de printemps ( 2 et 3 ) et une seule en culture d'été, la levée étant rapide et concentrée après semis. Le nombre d'épis de la céréale s'est montré par ailleurs un bon prédicteur de la survie des plantules au printemps (DEBAEKE, 1988b); l'action conjuguée classe d'âge-densité épis n'est cependant pas connue.

La compétition inter- et intra-spécifique n'est pas négligeable pour les graminées annuelles et pour les situations fortement infestées. En zone désherbée efficacement chaque année et pour des communautés où dominent les dicotylédones, cet effet est occasionnel et l'on admet que la répartition spatiale des plantes après traitement est aléatoire, sans recouvrement entre individus.

En résumé, les facteurs de variation du taux $\delta$ sont la nature et l'état de croissance de la culture d'une part, la période de levée de l'adventice d'autre part.

\section{Production de semences par plante}

La production semencière par plante adventice sous couvert d'une culture est très inférieure aux valeurs obtenues sur plantes isolées ou en sol nu (PAWLOWSKI et al., 1970). A la récolte, on trouve des plantes grenées, fleuries ou au stade végétatif; on distingue alors une production réelle (nombre de semences bien conformées) et une production potentielle (incluant également le nombre de semences issues de l'évolution après récolte des organes floraux présents à cette date), en supposant dans ce cas que la fructification est complète et le nombre de semences par fruit peu variable. On considère que seules les plantes ayant amorcé leur floraison et seules les fleurs présentes à la récolte peuvent produire des semences avant déchaumage; les plantes non fleuries à cette époque n'ont pas le temps de grener ou sont en cours de dégradation. La production effective se situe probablement entre ces deux pôles. Des semences enfouies avant maturité complète pourraient néanmoins évoluer en semences viables, ce qui atténue l'importance de la date de clôture du cycle. L'effet cohorte est moins prononcé que pour la survie des plantes, l'âge moyen des plantes présentes à la récolte étant assez homogène (DEBAEKE, 1987). Aussi la valeur de $p_{i+1}$ dépendra-t-elle uniquement de l'espèce et de la nature de la culture.

Comme les autres paramètres démographiques, $\mathrm{p}_{\mathrm{i}}$ est estimé en zone non désherbée; l'effet de l'herbicide ne se manifeste dans ce modèle que comme facteur de déclin de la densité de plantes établies. Les augmentations de production par plante observées après désherbage sont attribuées dans la littérature à une moindre compétition entre adventices (MüLLVERSTEDT, 1962; JoHNSTON \& COBLE, 1986 ; FERnANDEZ-QuinTANILLA et al., 1987); pour les raisons évoquées plus haut, on considère que cette compétition reste faible même en l'absence de désherbage, du moins en culture de céréales. On admet par ailleurs que la qualité des semences n'est pas affectée par l'herbicide. Les travaux existants montrent des pertes de viabilité et (ou) des réductions de dormance des semences produites en zone traitée (Peters et al., 1975; FAwCETT \& SLIFE, 1978 ; RAI \& TrIPATHI, 1986). Ces effets ne se manifestent que lorsque le désherbage se situe peu avant la floraison des adventices, ce qui les limite aux applications d'hormones tardives. Plus généralement, on admet que la capacité germinative des semences n'est pas affectée par l'histoire climatique ou culturale de la parcelle, ce qui est une approximation (CHADGEUF-HANNEL, 1985).

\section{Devenir des semences tombées au sol}

On estime que toutes les semences produites et tombées au sol $\left(\mathbf{P}_{i+1}\right)$ sont incorporées au stock $S_{i+1}$. Le devenir de ces organes après dissémination constitue en effet un chaînon manquant dans les études de dynamique, en raison des difficultés d'estimation inhérentes. Le 
caractère restreint des études abordant ce sujet (séjour prolongé des semences en surface; espèces prairiales; graminées à grosses semences) et la variabilité importante des pertes de semences, ne nous permettent pas d'apprécier clairement l'importance quantitative de ce poste (MARShall \& JAIN, 1970 ; SAGAR \& Mortimer, 1976; MORTIMER, 1979). Ce phénomène qui fait intervenir climat, pathogènes et prédateurs est probablement réduit en sol travaillé; le déchaumage, survenant souvent peu après libération des semences, permet de les protéger contre les diverses dégradations, comme l'ont montré Wilson \& Cussans (1975) pour Avena fatua. Dans le seul cas des graminées annuelles, des travaux ont montré que le ramassage des pailles de céréales ou leur brûlage provoquent des disparitions de semences en surface, moins importantes cependant que les quantités exportées avec la récolte (WILson \& Cussans, 1975; Moss, 1980).

\section{CONCLUSION GÉNÉRALE}

La figure 3 résume de manière synthétique les sources de variation et le mode d'estimation des divers paramètres, en distinguant sources bibliographiques et expérimentales, éléments de l'itinéraire technique et états de la végétation adventice ou cultivée, mesures annuelles ou simulations. La démarche proposée a permis de décrire, pas à pas et de manière déterministe, l'évolution pluriannuelle d'un stock semencier, sous l'effet combiné du

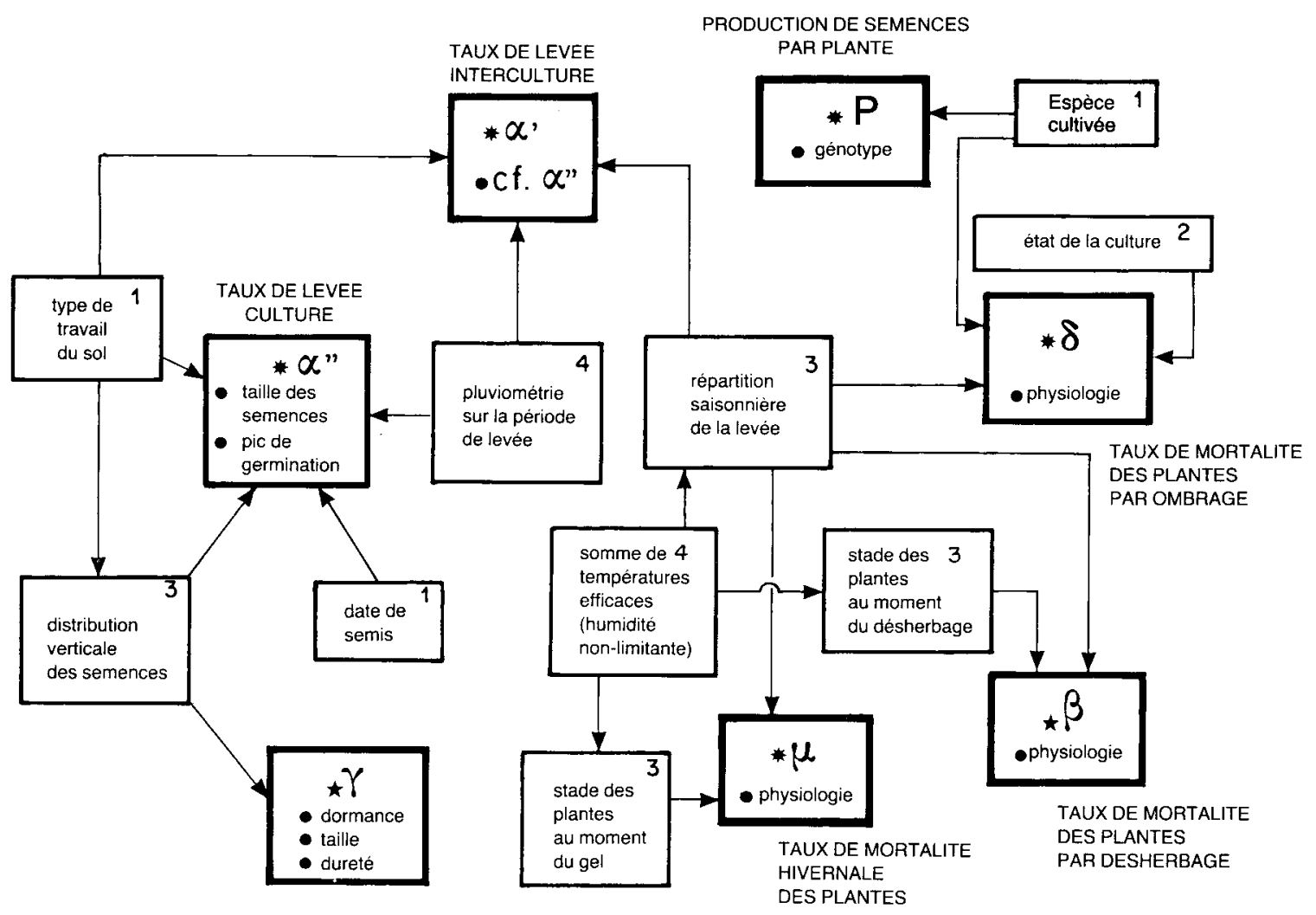

Figure 3

MORTALITE DES SEMENCES DANS LE SOL

Représentation schématique des facteurs de variation des paramètres pris en compte dans le modèle

1) Eléments de l'itinéraire technique.

2) Etats mesurés du peuplement cultivé

3) Etats de la flore adventice simulés par le modèle.

4) Données climatiques mesurées.

Effet espèce moyen: valeurs issues de la bibliographie (*) valeurs expérimentales $(\star)$.

Schematic representation of the factors acting on the parameters values in our model.

1) Elements of crop management.

2) Measured crop states.

3) Simulated weed states.

4) Measured climatic factors.

Mean species effect : bibliographic values (*) experimental values ( $\star$ ). 
climat et des techniques culturales. Les effets cumulatifs ont été pris partiellement en charge (distribution verticale des semences enfouies). Cependant ce modèle général mais simplifié s'applique, dans sa construction actuelle, aux espèces basses, à dissémination aérienne peu efficace, et aux densités d'infestation faibles (pas de compétition entre adventices, peu d'effet dépressif sur le rendement de la culture); ce type de flore caractérise les situations régulièrement et efficacement désherbées, où dominent les dicotylédones annuelles. Comme cela a été développé pour d'autres composantes de la fertilité (SEBILloTtE, 1982), cette approche permet une gestion quantitative du stock semencier, ignorant une modifi- cation progressive probable de la population étudiée (sélection de génotypes et donc changement de la valeur des paramètres démographiques) et l'introduction voire le développement d'espèces absentes à l'origine du bilan.

Reçu le 3 novembre 1987. Accepté le 29 mars 1988.

\section{REMERCIEMENTS}

Les auteurs remercient Messieurs Barralis et Perny qui ont accepté de lire ce manuscrit et de leur faire part de leurs critiques et conseils

\section{RÉFÉRENCES BIBLIOGRAPHIQUES}

Anonyme, 1983. Sensibilité des mauvaises herbes aux herbicides, A.C.T.A., Paris, 211 p.

Anonyme, 1987. Les herbicides des céréales. $N^{\circ}$ Spécial, Perspect. agric., I.T.C.F.

Archibold O. W., Hume L., 1983. A preliminary survey of seed input into fallow fields in Saskatchewan. Can. J. Bot., 61, 1216-1221.

Barralis G., 1982. La flore adventice des cultures et son évolution. Bull. tech., Inf., $\mathrm{N}^{\circ}$ Spécial «Fertilité du milieu et agriculture», 370-372, 463-466.

Barralis G., Chadoeuf R., 1976. Evolution qualitative et quantitative d'un peuplement adventice sous l'effet de 10 années de traitement. $5^{e}$ Col. Int. Ecol. Biol. Mauv. Herbes, Dijon, 179-186.

Barralis G., Chadoeuf R., 1980. Etude de la dynamique d'une communauté adventice. I. Evolution de la flore adventice au cours du cycle végétatif d'une culture. Weed Res., 20, 231-237.

Barralis G., Chadoeuf R., 1987. Potentiel semencier des terres arables. Weed Res., 27, 417-424.

Beuret E., 1984. Expression et évolution du stock grainier des sols; influence de l'assolement et de l'époque des travaux du sol. $7^{c} \mathrm{Col}$. Int Ecol. Biol. Syst. Mauv. Herbes, Paris, 81-90.

Chadoeuf R., Barralis G., Lonchamp J. P., 1984. Evolution du potentiel semencier de mauvaises herbes annuelles dans un sol cuitivé. $7^{e}$ Col. Int. Ecol. Biol. Syst. Mauv. Herbes, Paris, 63-70.

Chadoeuf-Hannel R., 1985. La dormance chez les semences de mauvaises herbes, Agronomie, 5 (8), 761-772.

Chancellor R. J., 1976. Changes in the weeds of upper Begbroke Field 1961-1976. 5* Col. Int. Ecol. Biol. Mauv. Herbes, Dijon, 227-234.

Cussans G. W., 1976. Population studies. In Wild oats in world agriculture. D. Price Jones Ed., A.R.C., London, 119-125.

Cussans G. W., Moss S. R., 1982. Population dynamics of annual grass weeds. Proc. Br. Crop Prot. Symp. on Decision Making in the Practice of Crop Protection, 91-98.

Debaeke P., 1987. Effets des systèmes de culture sur la flore adventice dicotylédone annuelle; intérêt de la modélisation pour l'étude de l'évolution à long terme du stock de graines de l'horizon travaillé. Thèse Doct. Ing. I.N.A. Paris-Grignon, 342 pages + annexes.

Debaeke P., 1988a. Dynamique de quelques dicotylédones adventices en culture de céréale. I. Relation flore levée - stock semencicr. Weed Res., (sous presse).

Debaeke P., 1988b. Dynamique de quelques dicotylédones adventices en culture de céréale. II. Survie, floraison et fructification. Weed Res. (sous presse).

Erviö L. R., 1977. The effect of sowing density and mecoprop treatment on competition between winter wheat and Triplearospermum inodorum (L.) Schultz Bip. Ann. agric. Fenn., 6, 110-116.

Fawcett R. S., Slife F. W., 1978. Effects of 2,4-D and dalapon on 'weed seed production and dormancy. Weed Sci., 26, 543-547.

Fernandez-Quintanilla C., Navarette L., Torner C., Andujar I. L., 1987. Influence of herbicide treatments on the population dynamics of Avena sterilis spp. ludoviciana (Durieu) Nyman in winter wheat crops. Weed Res., 27, 375-383.
Fogelfors H., 1977. The competition between barley and five weed species as influenced by MCPA treatment. Swed. J. agric. Res., 7, 147-151.

Froud-Williams R. J., Chancellor R. J., Drennan D. S. H., 1981 Potential changes in weed floras associated with reduced-cultivation systems for cereal production in temperate regions. Weed Res., 21 , 99-109.

Froud-Williams R. J., Chancellor R. J., Drennan D. S. H., 1984. The effects of seed burial and soil disturbance on emergence and survival of arable weeds in relation to minimal cultivation. J. appl. Ecol., 21, 629-641

Hammerton J. L., 1970. Crop seed-rate and MCPA effectiveness. Weed Res., 10, 178-180.

Harper J. L., 1977. Population biology of plants, Academic Press, London, $892 \mathrm{p}$.

Hurka H., Haase R., 1982. Seed ecology of Capsella bursa-pastoris Moench. : dispersal mechanisms and the soil seed bank. Flora, Jena, $172,35-46$

Johnston W. C., Coble H. D., 1986. Crop rotation and herbicide effects on the population dynamics of two annual grasses. Weed Sci., 26, 452-456.

Koch W., 1970. Temperature requirements of weeds for germination. Sautgut-Wirtschaft, 22, 85-86 (en allemand).

Leguizamon E. S., Roberts H. A., 1983. Seed production by an arable weed community. Weed Res., 22, 35-39.

Major J., Pyott W. T., 1966. Buried, viable seeds in two California bunch grass sites and their bearing on the definition of a flora. Vegetatio, Acta Geobot., 13, 253-282.

Manlove R. J., Mortimer A. M., Putwain P. D., 1982. Modelling wild oat populations and their control. Proc. $1982 \mathrm{Br}$. Crop Prot. Conf. Weeds, 749-756.

Marshall D. R., Jain S. K., 1970. Seed predation and dormancy in the population dynamics of Avena fatua and Avena barbata. Ecology, 51, 886-891.

Marty J. R., Hilaire A., Perny R. A., 1971. Quelques aspects du désherbage de la succession sorgho-blé dans la région toulousaine. C. R. Acad. Agr. Fr., 57, 1627-1637.

McRill M., Sagar G. R., 1973. Earthworms and seeds. Nature, London, 243, 482.

Montegut J., 1975. Ecologie de la germination des mauvaises herbes. In "La germination des semences ». R. Chaussat et $Y$. Le Deunff Eds. Gauthier-Villars, Paris, 191-217.

Mortimer A. M., 1979. The influence of cultural measures and herbicide practices on the fate of weed seeds. Proc. EWRS Symp. on the Influence of Different Factors on the Development and Control of Weeds, Mainz, 135-143.

Mortimer A. M., Putwain P. D., MeMahon D. J., 1978. A theoretical approach to the prediction of weed population sizes. Proc. $1978 \mathrm{Br}$. Crop Prot. Conf., Weeds, 415-474.

Mortimer A. M., McMahon D. J., Manlove R. J., Putwain P. D., 1980. The prediction of weed infestations and cost of differing control strategies. Proc. 1980 Br. Crop Prot. Conf., Weeds, 415-474. 
Moss S. R., 1980. Some effects of burning straw on seed viability, seedling establishment and control of Alopecurus myosuroides Huds., Weed Res., 20, 271-276.

Moss S. R., 1983. The production and shedding of Alopecurus myosuroides Huds. seeds in winter cereal crops. Weed Res., 23, 45-51.

Müllverstedt R., 1962. Vergleich der Auswirkung mechanischer auf Massenwachstum und Samenproduktion der verbliebenen Unkrauter. Z. Pflanzenkr. Pflanzenschutz Sonderh., 73, 598-603.

Pawlowski F., Malicki L., 1968. Effect of different methods of ploughing on vertical distribution of weed seeds in soil formed of loess. Ann. Univ. Mariae Curie Sklod., Sect. E, 23, 161-174 (en polonais).

Pawlowski F., Wszolek M., 1978. Weediness of the summer barley and winter wheat grain on loesses and chernozems in the Hrubieszow region. Rocz. Nauk Rolnic., A, Prod. Rosl., 103, 131-145 (en polonais).

Pawlowski F., Kapelusny J., Kolasa A., Lecyk Z., 1970. The prolificacy of weeds on stubble fields in the Lublin Voivodeship. Ann. Univ. Mariae Curie Sklod., Sect. E, 25, $49-59$ (en polonais).

Peters N. C. B., Chancellor R. J., Dreman D. S. H., 1975. Dormancy of seed from wild oat plants sprayed with sub-lethal levels of herbicides and gibberellic acid. Proc. EWRS Symp. on Status, Biology and Control of Grassweeds in Europe, Paris, 87-94.

Pollard F., 1982. A computer model for predicting changes in a population of Bromus sterilis in continuous winter cereals. Proc. 1982 Br. Prot. Conf., Weeds, 973-979.

Popay A. I., Roberts E. H., 1970. Ecology of Capsella bursa-pastoris and Senecio vulgaris in relation to germination behaviour. J. Ecol., 58, 123-139.

Psarski P., Metreau D., Le Roux A., 1986. La levée au champ des adventices dans les rotations. $13^{e}$ Conf. ANPP-COLUMA, Versailles, $37-53$.

Rademacher B., 1964. Auswirkungen des Kahlfrost-Winters 1961-62 auf Bestand Bekämpfung der überjährigen Unkrauter in Winterungen. Z. Pfanzenkr. Pfanzenschutz, Sonderh., 2, 35-40.

Rai J. P. N., Tripathi R. S., 1986. Population regulation of Galinsoga ciliata and $G$. parviffora: effect of 2 4-D application at different growth stages and light regimes. Weed Res., 26, 59-67.

Rauber R., Koch W., 1975. Zur Populationdynamik des Flughafers (Avena fatua L.) unter dem Aspekt der langfristigen Befallsprognose. Proc. EWRS Symp. Status and Control of Grassweeds in Europe, Paris, 113-123.

Roberts H. A., 1963. Studies on the weeds of vegetable crops. III. Effect of different primary cultivations on the weed seeds in the soil. J. Ecol., 51, 83-96.

Roberts H. A., 1970. Viable weed seeds in cultivated soil. Rep. Natn. Veg. Stn. for 1969, 25-38.

Roberts E. H., 1972. Dormancy : a factor affecting seed survival in the soil. In "Viability of seeds", E. H. Roberts Ed., Chapman and Hall, London, 321-359.

Roberts H. A., 1981. Seed banks in soil. Adv. appl. Biol., 6, 1-55.
Roberts H. A., Chancellor R. J., 1986. Seed banks of some arable soils in the English midlands. Weed Res., 26, 251-301.

Roberts H. A., Dawkins P. A., 1967. Effect of cultivation on the number of viable weed seeds in soil. Weed Res., 7, 290-301.

Roberts H. A., Feast P. M., 1973. Emergence and longevity of seeds of annual weeds in cultivated and undisturbed soil. J. appl. Ecol., 10, 133-143.

Roberts H. A., Potter M. E., 1980. Emergence patterns of weed seedlings in relation to cultivation and rainfall. Weed Res., 20, 377-386.

Robinson E. L., Kust C.A., 1962. Distribution of witchweed seeds in the soil. Weeds, 10, 335.

Sagar G. R., Mortimer A. M., 1976. An approach to the study of the population dynamics of plants with special reference to weeds. Appl. Biol., 1, 1-47

Salisbury E. J., 1961. Weeds and Aliens, Collins, London, 384 p.

Schweizer E. E., Zimdahl R. J., 1984. Weed seed decline in irrigated soil after rotation of crops and herbicides. Weed Sci., 32, 84-89.

Sebillotte M., 1982. Pratiques des agriculteurs et évolution de la fertilité du milieu. Eléments pour un jugement des systèmes de culture. Bull. tech. Inf., $\mathrm{N}^{\circ}$ Spécial «Fertilité du milieu et agriculture», 370-372, 425-436.

Selman M., 1970. The population dynamics of Avena fatua (wild oats) in continuous spring barley. Desirable frequency of spraying with triallate. Proc. 10th Br. Weed Cont. Conf., 1176-1184.

Sobey D. G., 1981. Biological flora of the British Isles. 150. Stellaria media. J. Ecol., 69, 311-335.

Stoller E. W., Wax L. M., 1974. Dormancy changes and fate of some annual weed seeds in the soil. Weed Sci., 22, 151-155.

Taylorson R. B., 1970. Changes in dormancy and viability of weed seeds in soils. Weed Sci., 18, 265-269.

Tonkin J. H. B., Phillipson A., 1973. The presence of weed seeds in cereal seed drills in England and Wales during spring 1970. J. natl. Inst. agric. Bot., 13, 1-8.

Walker A., Barnes A., 1981. Simulation of herbicide persistence in soil : a revised computer model. Pestic. Sci., 12, 123-128.

Wellington P. S., 1960. Assessment and control of the dissemination of weeds by crop seeds. In "The Biology of Weeds", J. L. Harper Ed., Blackwell Scientific Publihers, Oxford, 94-107.

Wilson B. J., 1970. Studies of the shedding of seed of Avena fatua in various cereal crops and the presence of this seed in the harvested material. Proc. 10th Br. Weed Cont. Conf., 831-836.

Wilson B. J., Cussans G. W., 1975. A study of the population dynamics of Avena fatua L. as influenced by straw burning, seed shedding and cultivations. Weed Res., 15, 249-258.

Wilson B. J., Cousens R., Cussans G. W., 1984. Exercices in modelling populations of Avena fatua L. to aid strategic planning for the long term control of this weet in cereals. $7^{e} \mathrm{Col}$. Ecol. Biol. Syst. Mauv. Herbes, Paris, 287-294. 